\title{
Posterior Tibial Tendon Transfer for Central Foot Drop: A Case Report
}

\author{
John Butarbutar*(D), Mirna Phandu, Lasa Dhakka Siahaan (D), Prettysia Suvarly (D) \\ Department of Orthopedics and Traumatology, Faculty of Medicine, Universitas Pelita Harapan, Siloam Hospitals Lippo Village, \\ Tangerang, Indonesia
}

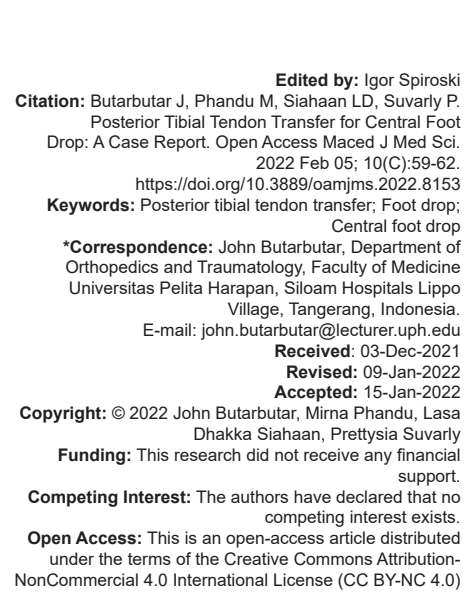

Introduction

Foot drop is a deformity that leads to severe restrictions in quality of life and impairment of daily activities, primarily when walking [1]. With foot drop, during the swing phase of walking, extra flexion of the hip and knee and ipsilateral elevation of the hip are needed to lift the foot adequately [2]. This gait is described as a high steppage gait [3]. High steppage gait consumes more energy than a normal gait and increases the risk of fall while walking [3]. Peripheral causes of foot drop are widely known. However, causes stemming from the central nervous system are rare [4]. One of the surgical intervention treatments for foot drop is posterior tibial tendon (PTT) transfer [1], [2], [3]. PTT transfer has been widely used for treating peripheral causes of foot drop but not central causes [1], [2], [3]. We report a case of central foot drop (CFD) that was corrected with PTT transfer. This article has followed SCARE checklist and guidelines and consent was given to our patient regarding the data obtained in this case would be submitted for publication [5].

\section{Case Report}

A 68-year-old female came to our outpatient department presented with progressive weakness and numbness on both of her feet. She complained that she was unable to walk normally because of the condition of her feet. The patient had a history of trauma approximately 1 year before admitting to our hospital. She is also on treatment for Parkinson disease. Physical examination revealed a high steppage gait when walking with a walker. Foot drop is seen on both of her feet. Babinski and clonus were positive on examination.

Lumbar X-ray and whole-spine magnetic resonance imaging $(\mathrm{MRI})$ were obtained after the trauma, which showed a fracture compression of the $12^{\text {th }}$ thoracic vertebrae and spinal stenosis at the $12^{\text {th }}$ thoracic level (Figures 1 and 2). Based on the physical examination and MRI, the patient was diagnosed with a central foot drop and underwent PTT transfer for the left foot.

The patient was placed in the supine position on the surgical table under spinal anesthesia, and a 


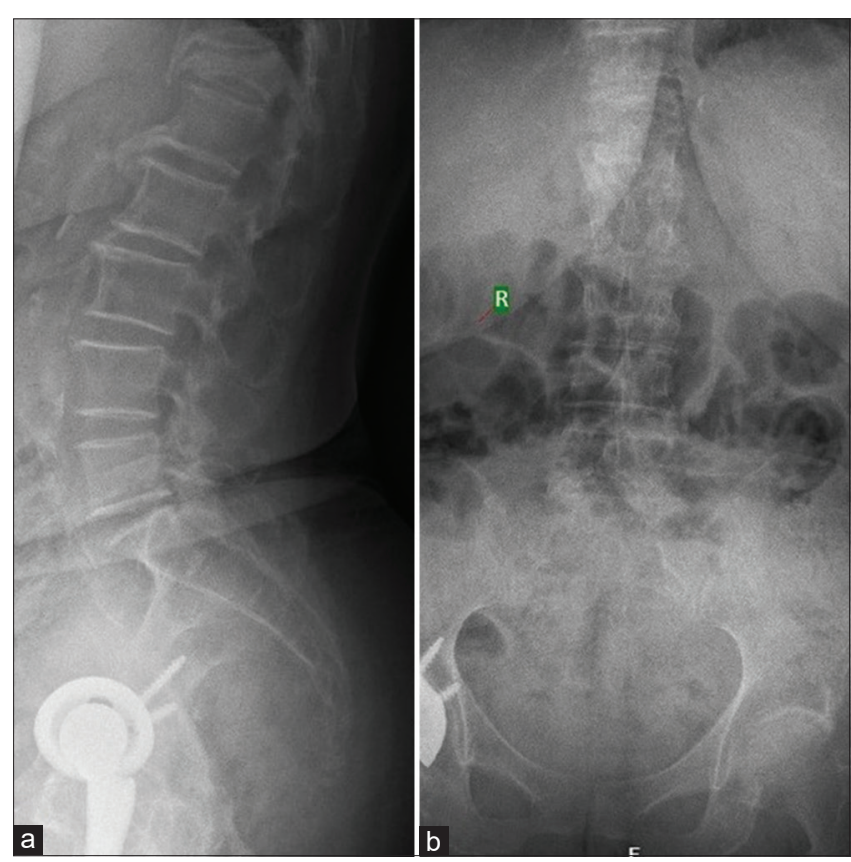

Figure 1: ( $a$ and b) Anteroposterior(AP)/lateral X-ray examination

pneumatic tourniquet was applied. A posteromedial incision is made just distal to the medial malleolus over the posterior tibialis tendon, and the tendinous attachment on the navicular is dissected subperiosteally (Figure 3a). A second $5 \mathrm{~cm}$ incision was made just posterior to the posteromedial border of the tibia then the posterior tibialis tendon was identified and mobilized proximally (Figure $3 \mathrm{~b}$ ). About $15 \mathrm{~cm}$ proximal to the ankle joint laterally, a $5 \mathrm{~cm}$ anterolateral incision is made, and the interosseous membrane was incised with tissue scissors along the tibial cortex. The interosseous membrane should be widely opened to prevent adhesions to the tibia and improve lateral lift. A $3 \mathrm{~cm}$ longitudinal incision is made overlying the navicular on the dorsal surface of the foot and bone tunnel was made by drilling (Figure 3c). The posterior tibialis tendon is transferred from the posterior to the anterior compartment through the interosseous membrane. Then, the posterior tibialis tendon was transferred
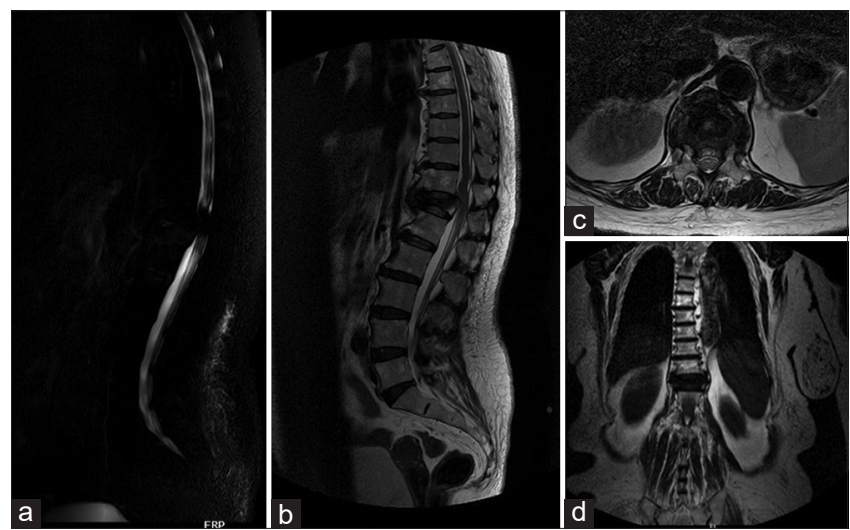

Figure 2: Lumbar magnetic resonance imaging (MRI) examination (a) Myelography. (b) T2 sequence MRI lumbar sagittal plane. (c) T2 sequence MRI lumbar axial plane. (d) T2 sequence MRI lumbar coronal plane through the dorsal foot incision subcutaneously. The posterior tibialis tendon was attached to the predetermined distal attachment with an interference screw while keeping the foot dorsiflexed slightly beyond neutral (Figure 3d). The foot must be balanced and in a neutral position at the end of the procedure, and a below-knee plaster was applied. The patients were immobilized in a cast with no weight-bearing for 6-8 weeks before initiating physical therapy without passive plantar flexion. The patients are retained in an ankle foot orthosis (AFO) for the first 3 months at night. Three months follow-up after the PTT transfer procedure, there is an improvement on the gait (during the swing phase of the affected foot, the patient does not require to lift the foot as high as before) and the foot drop is corrected with the American Orthopaedic Foot and Ankle Society (AOFAS) score 68\%.

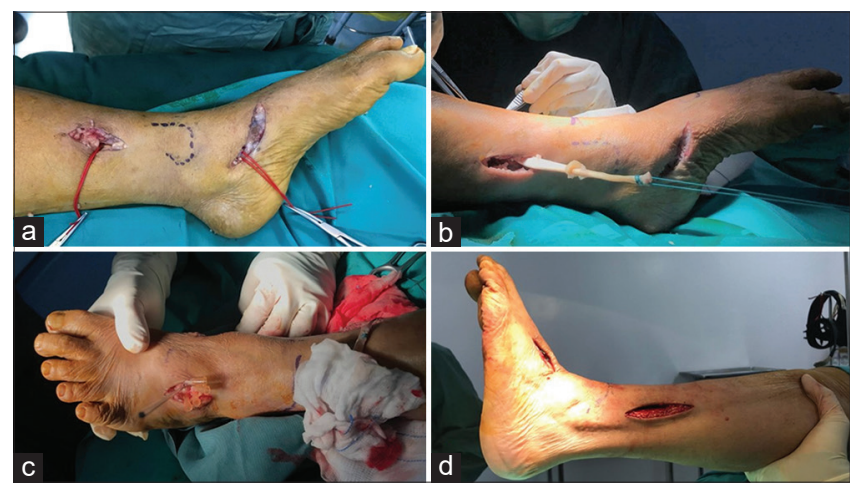

Figure 3: (a) A posteromedial incision is made just distal to the medial malleolus over the posterior tibialis tendon, and the tendinous attachment on the navicular is identified. (b) The posterior tibialis tendon was identified and mobilized proximally through a second incision posterior to the posteromedial border of the tibia. (c) $A 3 \mathrm{~cm}$ longitudinal incision is made overlying the navicular on the dorsal surface of the foot and bone tunnel was made by drilling. (d) The posterior tibialis tendon was attached while keeping the foot dorsiflexed

\section{Discussion}

Foot drop is described as an inability to do dorsiflexion on foot. This deformity is caused by weakness of the anterior tibialis muscle and frequently accompanied by weakness of the extensor hallucis longus and extensor digitorum longus [2]. Foot drop deformity can be caused by neurologic causes such as central or peripheral neurogenic lesion and muscular causes [1], [2]. Peripheral causes of foot drop are common but central causes are rare [4]. Peripheral and central causes are differentiated by the location of the lesion causing the foot drop; central causes foot drop caused by lesion along the UMN tract, which is from the brain through spinal cord until L4-L5 level [6]. In this case, the patient has an old trauma that caused a fracture compression on the $12^{\text {th }}$ thoracic vertebrae and spinal stenosis at the $12^{\text {th }}$ thoracic level. 
Patients with foot drop have an abnormal gait cycle. During the swing phase of walking, extra flexion of the hip and knee and ipsilateral elevation of the hip is needed to lift the foot adequately as the foot could not actively dorsiflex. This gait is described as a high steppage gait observed on the patient when walking [3].

Eskandry, in his study, described that there are foot drop patients with central causes but with a clinical manifestation resembling foot drop with peripheral causes (e.g., sciatic nerve injury), which can be differentiated with Babinski sign or hyperactive ankle jerk or clonus [7]. Tokuhashi et al. found that two of three subjects with the $12^{\text {th }}$ thoracic $-1^{\text {st }}$ lumbar disc herniation had symptoms of bilateral foot drop and one unilateral foot drop [8]. This study showed that, although rare, central causes foot drop clinical manifestation can resemble peripheral causes foot drop which seen on this patient.

The aim of therapy for foot drop is to restore the foot's dorsiflexion and restore normal gait [2]. Ankle brace may correct the high steppage gait and prevent the patient from falling, but being dependent on orthotic ankle braces means a reduction in quality of life, and apart from esthetic reasons, ankle braces increase the risk of recurrent skin lesion [1]. Other management include nerve repair and nerve stimulus [1]. Surgical treatment option for foot drop consists of tenodesis, arthrodesis, and tendon transfer. Surgical treatment should be recommended to patients who would prefer to discard ankle braces [9]. Tendon transfer represents the gold standard for restoring dorsiflexion on foot drop because the transfer of posterior tibial tendon restores the dorsiflexion of the foot and removes the deforming force on the medial aspect of the foot [2], [3], [10]. In tendon transfer, normal gait cycle is theoretically possible [3]. Because to achieve a normal gait cycle, other than the ankle should be able to actively dorsiflexed, the ankle is required to reach $10^{\circ}$ of dorsiflexion and should be able to passively bent $10-26^{\circ}$ in plantar flexion [3]. In ankle arthrodesis and tenodesis, the recommended angle of the foot is neutral [3]. Thus, a normal gait is impossible to achieve in patients with ankle arthrodesis and tenodesis [3]

Before we decide to use tendon transfer, the posterior tibial tendon must be sufficiently powerful because when a tendon is rerouted to a new insertion point, its muscle power will decrease at least one grade [3]. This is important because if the posterior tibial muscle is not sufficiently powerful, active dorsiflexion after the procedure will not be achieved and function as a tenodesis only. In this case, the posterior tibial muscle was assessed, and the muscle power was five.

Ober first described the posterior tibial tendon transfer in 1933 [2]. Ober transferred the posterior tibial tendon anteriorly through the circumtibial route and fixed it to the third metatarsal bone [2]. Then, in 1954, Watkins made the same posterior tibial tendon transfer but through interosseous route, and both techniques are reported to be successful in the surgical treatment for foot drop [2]. Recent studies reported that even though it is more technically demanding, the intraosseous route is more efficient compared to the circumtibial or subcutaneous route because this technique is more physiologic from the viewpoint of direction, more dorsiflexion power, minimal pronation, and a better esthetic result compared to subcutaneous route [1], [2], [9], [11]. Despite subcutaneous route has less power in dorsiflexion, Soares's study shows that both methods have a good outcome and achieve above neutral dorsiflexion in $80 \%$ of the patients [2]. Soares, in his study, reported too that circumtibial route cases were associated with a high rate of recurrent inversion caused by ulceration of the lateral border of the foot. In his study, the circumtibial route was reserved for patients with a calcified and unyielding intraosseous membrane [12].

When transferring the tendon through the intraosseous route, the window in the intraosseous membrane must not be too narrow because adhesions are likely to form later after the procedure [13]. Yeap, in his study, reported that early passive movement after the tendon transfer procedure through the intraosseous route might reduce adhesions and improve the outcome of the surgery [9].

The posterior tibial tendon has only $2 \mathrm{~cm}$ of sliding amplitude compared to the anterior tibial tendon with about 3-5 cm sliding amplitude [1], [13]. Therefore, posterior tibial tendon transfer must be fixed at the anterolateral part of the foot under high tension [2], [13]. Studies reported that patients have inadequate active dorsiflexion due to inadequate tension at the transfer so that the tension of the transfer must be adjusted cautiously [2], [13]. Soares, in his study, reported that between the time of tendon transfer and discharge from the hospital, there was a $10^{\circ}$ decrease in dorsiflexion and a further $5-10^{\circ}$ decrease during follow-up. Because of that, he suggested keeping the ankle at $20^{\circ}$ dorsiflexion during the tendon transfer [2].

Passive dorsiflexion should be assessed before the tendon transfer procedure and should be at least $10^{\circ}$ beyond neutral [1], [9]. Hove, in his study, recommends that at least passive dorsiflexion, $20^{\circ}$ beyond neutral is achieved before the procedure [13]. Pre-operative training program such as exercises for the tendon to be transferred and stretching exercises of the Achilles tendon is recommended to achieve better passive dorsiflexion. If the foot could not passively dorsiflex $20^{\circ}$ beyond neutral, Achilles tendon lengthening is recommended [13].

The tendon transfer insertion site could be tendon to bone or tendon to tendon fixation [2], [11]. The insertion site can be fixed using a tunnel opened in the tarsal or metatarsal bones [2]. The fixation point should be considered based on deformity present at the foot. The shift of the fixation point medially or laterally may result in valgus or varus deformities [2], [9]. 
Chuan, in his study, chooses the insertion point for posterior tendon transfer at intermediate cuneiform or navicular bone in 31 patients. The navicular bone may be damaged due to posterior tibial tendon transfer to the navicular bone; however, long-term effects have not been clarified, and avascular necrosis of the navicular bone was not reported in all 31 patients in Chuan's study [3]. Extensors of all toes are also paralytic in tendon to bone fixation point, but clinically, it does not show significant disturbance for the patient [3].

Long-term loss of movement in the anterior tibialis muscle can lead to tendon atrophy. Choosing the anterior tibial tendon as an insertion point in tendon to tendon fixation induces a risk of anterior tibial tendon tear. In addition, tendon suture healing might not be favorable due to uncertain local blood supply. Therefore, the failure rate is increased in tendon to tendon fixation [3].

Due to the tendon transfer procedure, the absence of the posterior tibial tendon at the plantar surface does not cause any significant disability [14]. Posterior tibial tendon dysfunction has been known to cause acquired flatfoot deformity, but none in posterior tibial tendon transfer studies reported acquired flatfoot deformity after the procedure [1], [3], [9].

\section{Conclusion}

PTT transfer is a possible option to correct CFD. It is less invasive and has a good outcome. PTT transfer was considered because it is less invasive, and tenodesis or ankle arthrodesis still could be performed later if the PTT transfer outcome is not favorable.

\section{References}

1. Steinau HU, Tofaute A, Huellmann K, Goerts O, Lehnhardt M, Kammler J, et al. Tendon transfers for drop foot correction: Long-term results including quality of life assessment, and dynamometric and pedobarographic measurements. Arch Orthop Trauma Surg. 2011;131(7):903-10. https://doi. org/10.1007/s00402-010-1231-z

PMid:21246379

2. Turker O, Serdar T, Karahman O, Atakan A, Safiye O. Surgical restoration of drop foot deformity with tibialis posterior tendon transfer. Acta Orthop Traumatol Turc. 2007;41(4):259-65. PMid:18180553

3. Chi-Chuan W, Ching-Lung T. Anterior transfer of tibialis posterior tendon for treating drop foot: Technique of enforcing tendon implantation to improve success rate. Acta Orthop Belg. 2015;81(1):147-54.

PMid:26280868

4. Westhout F, Pare L, Linskey ME. Central causes of foot drop: Rare and underappreciated differential diagnoses. J Spinal Cord Med. 2007;30(1):62-6. https://doi.org/10.1080/10790268. 2007.11753915 PMid:17385271

5. Agha RA, Franchi T, Sohrabi C, Mathew G, Kerwan A, SCARE Group. The scare 2020 guideline: Updating consensus surgical case report (SCARE) guidelines. Int J Surg. 2020;84:226-30. https://doi.org/10.1016/j.ijsu.2020.10.034 PMid:33181358

6. Vigasio A, Marcoccio I, Patelli A, Mattiuzzo V, Prestini G. New tendon transfer for correction of drop-foot in common peroneal nerve palsy. Clin Orthop Relat Res. 2008;466:1454-66. https:// doi.org/10.1007/s11999-008-0249-9

PMid: 18414961

7. Eskandary H, Hamzei A, Yasamy AT. Foot drop following brain lesion. Surg Neurol. 1995;43(1):89-90. https://doi. org/10.1016/0090-3019(95)80048-I

PMid:7701434

8. Tokuhashi $\mathrm{Y}$, Matsuzaki $\mathrm{H}$, Uematsu $\mathrm{Y}$, Oda $\mathrm{H}$ Symptoms of thoracolumbar junction disc herniation. Spine (Phila Pa 1976). 2001;26(22):E512-8. https://doi. org/10.1097/00007632-200111150-00021 PMid:11707722

9. Yeap JS, Birch R, Singh D. Long-term results of tibialis posterior tendon transfer for foot-drop. Int Orthop. 2001;25(2):114-8. https://doi.org/10.1007/s002640100229

PMid:11409449

10. Ashraf A, Wael A, Hashem EF. Tibialis posterior tendon transfer for correction of drop-foot incommon peroneal nerve palsy. Egypt Orthop J. 2018;53(1):54-49.

11. Goh JC, Lee PY, Lee EH, Bose K. Biomechanical study on tibialis posterior tendon transfer. Clin Orthop Relat Res. 1995;(319):297-302.

PMid:7554643

12. Soares D. Tibialis posterior transfer for the correction of foot drop in leprosy. Long-term outcome. J Bone Joint Surg. 1996;78(1):61-2. PMid:8898128

13. Hove Leiv $M$, Nilsen Per T. Posterior tibial tendon transfer for drop foot: 20 Cases followed for 1-5 years. Acta Orthop Scand. 1998;69(6):608-10. https://doi. org/10.3109/17453679808999265

PMid:9930107

14. Yeganeh A, Motaghi A, Shahhoseini G, Farahini H. New method for fixation point of tibialis posterior tendon transfer. Med $\mathrm{J}$ Islam Repub Iran. 2013;27(4):163-7. 